www.jmscr.igmpublication.org ion
Impact Factor 3.79

ISSN (e)-2347-176x

crossref DOI: _http://dx.doi.org/10.18535/jmscr/v3i8.68

\title{
Metastatic Malignant Melanoma to Bone Marrow with Occult Primary - A Case Report
}

\author{
Authors \\ Krutika R. Bhatt ${ }^{1}$, Biren B. Parikh ${ }^{2}$, Priti P. Trivedi ${ }^{3}$, Manoj J. Shah ${ }^{4}$ \\ Gujarat Cancer and Research Institute, Ahmedabad, Gujarat, India \\ Email:dr.krutika25@gmail.com
}

\begin{abstract}
Background: Metastases of malignant melanoma to the bone marrow are very rare. A few case reports are published in the literature with a known primary site. Herein we report a case of metastatic malignant melanoma in bone marrow with occult primary.

Case: A 55-year-old-male presented with complains of bone pain. X ray and MRI hip revealed osteoporotic changes with multiple altered hypointensity lesions. Bone marrow aspirate and biopsy suggested metastatic malignant melanoma, which was confirmed by immunohistochemistry. No primary site was identified and it remained occult until patient developed blurring of vision 2 months later the primary diagnosis. That time possibility of choroidal melanoma was given considering the initial diagnosis on bone marrow. Patient died 3 months post diagnosis.

Conclusion: Malignant melanoma is a notorious neoplasma which primarily may present directly as distant metastasis with aggressive behavior and poor prognosis. Clinicians must be aware of the varied clinical manifestations of disseminated malignant melanoma even if the primary site is not evident.

Key words: Melanoma, bone marrow metastasis, occult primary
\end{abstract}

\section{Introduction}

Malignant melanoma accounts for $1-3 \%$ of all malignancies with an increasing incidence being seen worldwide ${ }^{[1]}$. Metastatic melanoma usually involves draining lymph nodes and occasionally adjacent skin first, but eventually metastasizes to distant visceral sites. The lung is most commonly involved followed by brain, liver, bone marrow, and intestine ${ }^{[2]}$. Metastases of melanoma to bone marrow are rare with widespread dissemination occurring in only $5-7 \%$ of cases ${ }^{[3]}$. A search of literature revealed only 34 cases of malignant melanoma metastasizing to bone marrow published till date. Out of which only 4 cases are reported as bone marrow metastasis with occult primary ${ }^{[4]}$. In about $5-15 \%$ of cases, metastatic melanoma is detected in the absence of an identifiable primary tumor. In these cases it is generally believed that the primary tumor has regressed ${ }^{[5]}$. Herein we report a case of malignant melanoma metastasized to bone marrow with an occult primary site.

\section{Case Report}

A 54 year old farmer presented with history of fall down followed by pain in left hip joint, back 
and shoulder and weakness of left leg. Symptomatic treatment was given but was not relieved. After thorough history and physical examination, all routine investigations were done. X-ray hip joint showed osteoporotic changes. MRI pelvis with bilateral hip joints and whole spine showed multiple large altered hypointensity lesions involving acetabular roof, iliac bone, pubis, ischium, sacrum, neck and upper shaft of femur and cervico-dorso-lumbar vertebrae suggesting the possibilities of lymphoproliferative neoplasms or multiple myeloma with differential diagnosis of lymphoma or metastatic lesion. [Figure 1] Serum levels of Carcinoembryonic Antigen (CEA) and Prostate Specific Antigen (PSA) were within normal limits.

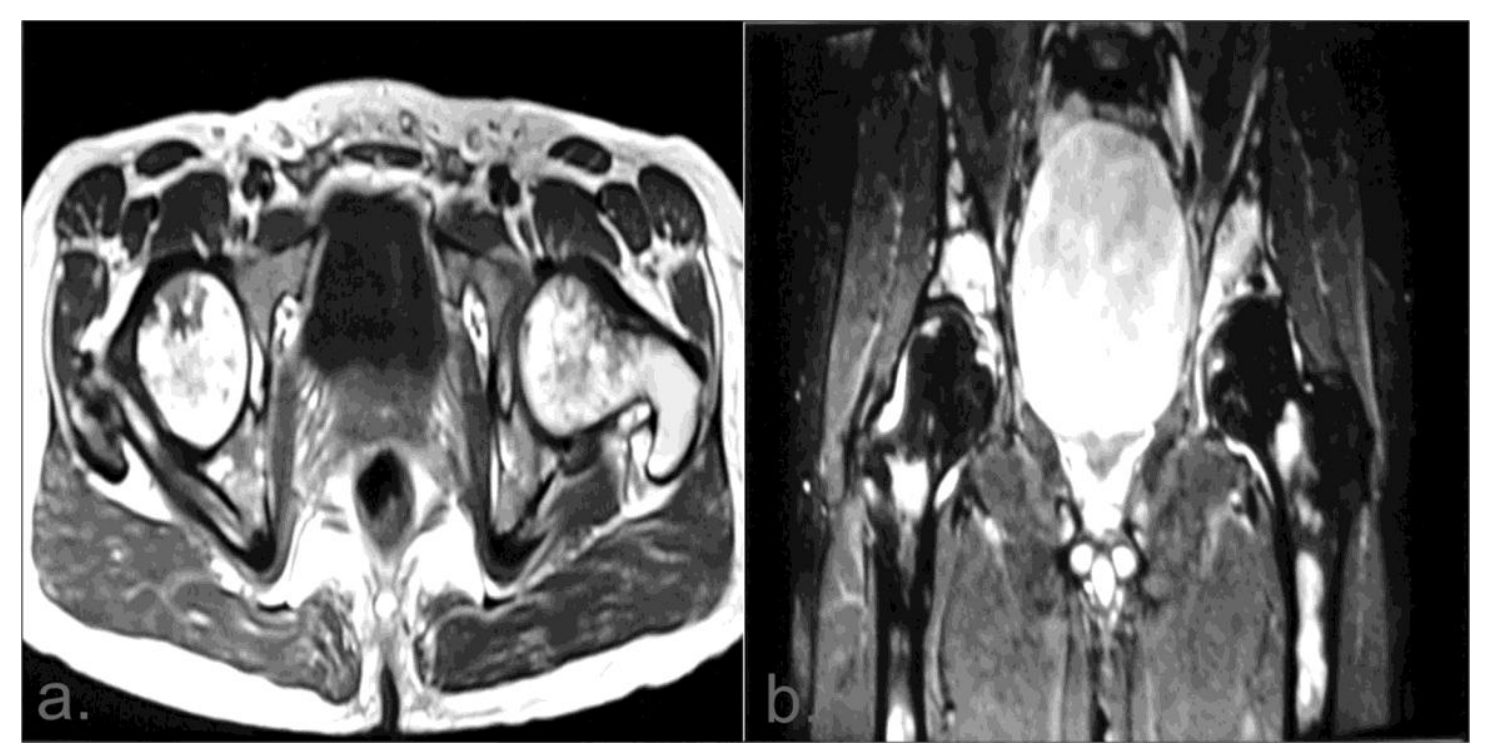

Figure 1 : a. and b. : MRI Pelvis with Bilateral Hip Joints showing hypointense lesions

Bone marrow aspirate (BMA) was dry. Bone marrow biopsy (BMBx) from left posterior superior iliac spine was hypercellular and showed many large atypical cells, arranged in sheets and clusters, which were pleomorphic with vesicular chromatin, prominent nucleoli and dense brown- black colored intracytoplasmic pigment in many cells along with few lymphocytes and erythroid precursors suggestive of metastatic malignant melanoma.[Figure 2] Fontana-Masson stain and melanin bleach confirmed the melanin nature of pigment.

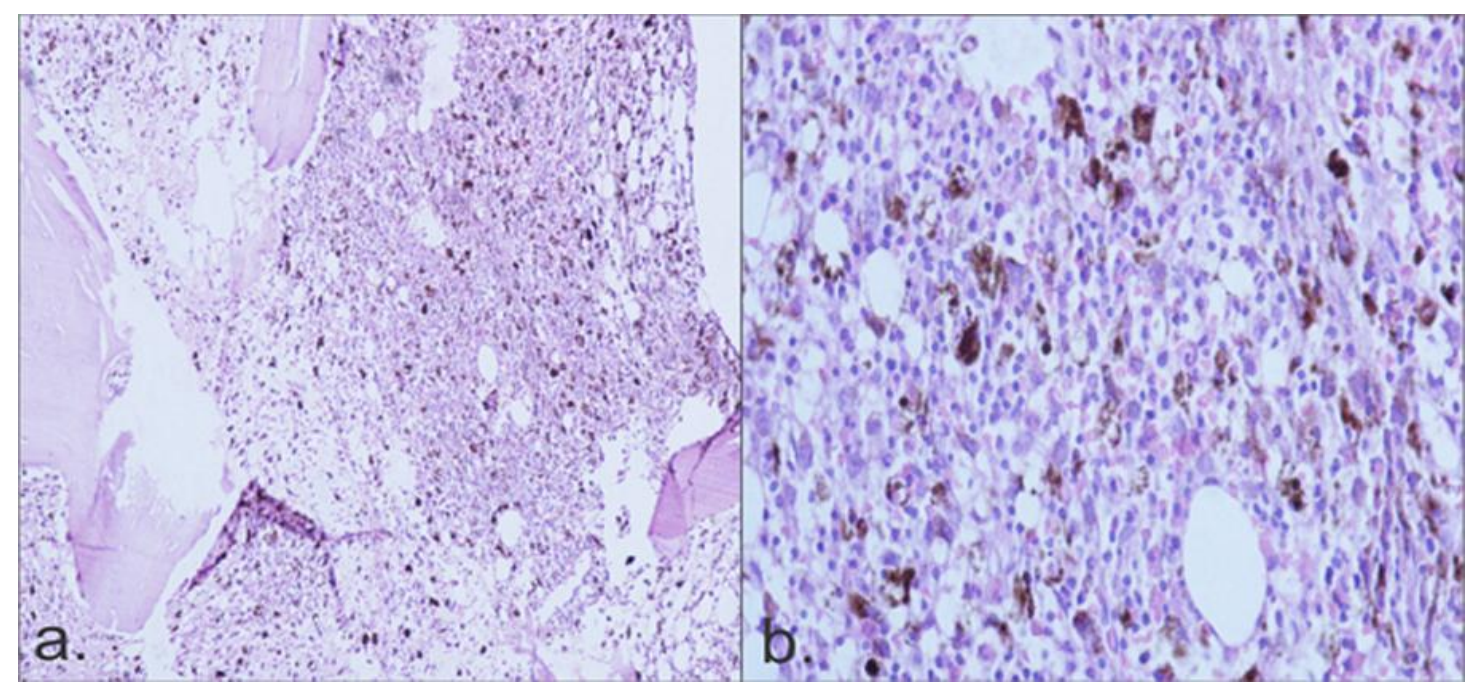

Figure 2 : a. low power (10x) and b. high power (40x) - bone marrow biopsy showing pigmented atypical cells of melanoma 
Immuno histochemistry (IHC) showed positivity for HMB-45, S-100, Vimentin and negativity for CD68 confirming the morphological diagnosis of Metastatic malignant melanoma.[Figure 3]

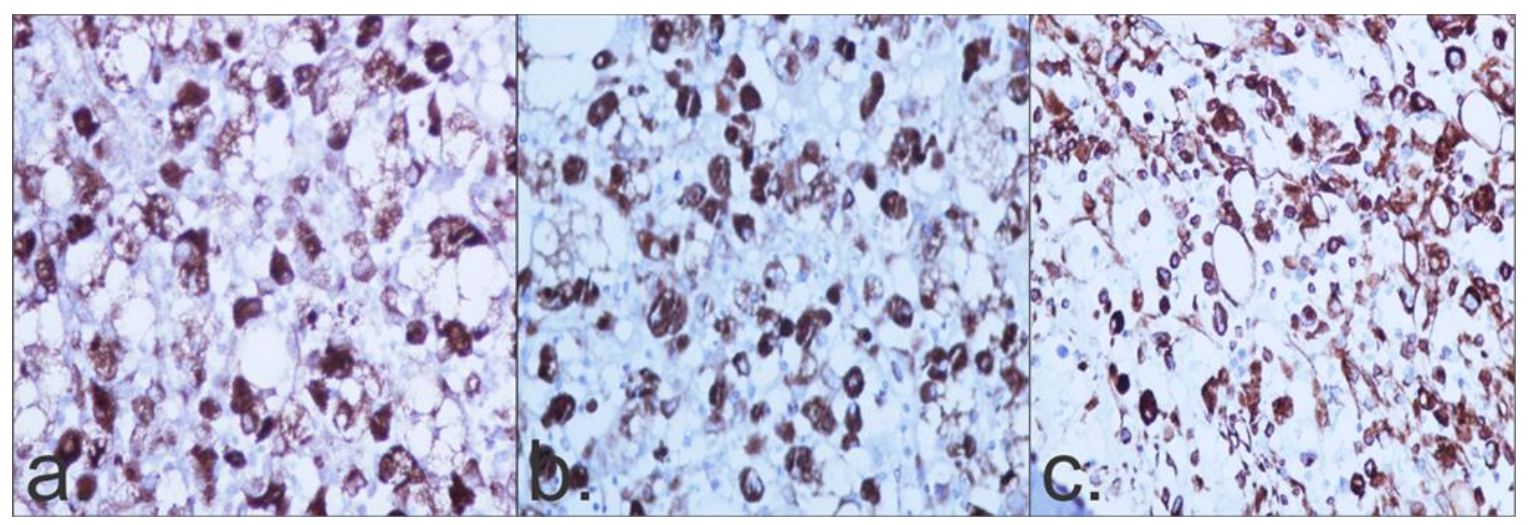

Figure 3 : a. HMB 45 - positive, b. S -100-positive, c. Vimentin - positive

2 months after the bone marrow and IHC report, patient complained of diminished vision since few days, so was referred to an ophthalmologist. After clinic - pathological correlation, possibility of choroidal melanoma was given on right eye $\mathrm{B}$ scan, which could be either primary or secondary. Patient was advised three cycles of Temozolamide (250mg) OD, for 5 days, at the interval of 21 days. But patient died 3 months post diagnosis before completing the treatment.

\section{Discussion and Conclusion}

When skeletal metastasis from malignant melanoma occurs, it is a sign of advanced stage of the disease. Metastasis to bone is stage IV according to American Joint Committee on Cancer [AJCC] for malignant melanoma and it usually occurs in disseminated disease. But in our case, most common sites of melanoma metastasis; liver, spleen, lungs were spared, with first presentation in bone marrow, with unknown primary site. Very handful cases are noted in literature with such entity. Prognosis ranges from 3 to 7 months in such cases. Bone biopsy and IHC have key role in diagnosing such rare entity like metastatic malignant melanoma to bone, sparing other organs. ${ }^{[6]}$ Bone marrow metastasis can be an initial presentation of malignant melanoma in otherwise healthy looking individual with bone pains.

\section{References}

1. Rigel DS, Friedman RJ, Kopf AW. The incidence of malignant melanoma in the United States: issues as we approach the 21st century. J Am Acad Dermatol 1996;34:839-47.

2. Monte SM, Moore GW, Hutchins GM. Patterned distribution of metastases from malignant melanoma in humans. Cancer Res 1983;43(7):3427-33.

3. Savage RA, Lucas FV, Hoffman GC. Melanoma in marrow aspirates. Am J Clin Pathol 1983;79:268-69.

4. Jain D, Singh T, Kumar N, Daga MK. Metastatic malignant melanoma in bone marrow with occult primary site - a case report with review of literature. Diagnostic Pathology 2007;2:38

5. Anbari KK, Schuchter LM, Bucky LP, Mick R, Synnestvedt M, Guerry D et al. Melanoma of unknown primary site: presentation, treatment, and prognosis - a single institution study. University of Pennsylvania Pigmented Lesion Study Group. Cancer 1997;79(9):1816-21.

6. Yaziji H, Gown AM. Immunohisto chemical markers of melanocytic tumors. Int J Surg Pathol 2003;11(1):11-15. 